\title{
The centralized energy supply in a network of distributed energy systems: A cost-based mathematical approach
}

\author{
Alberto Fichera, Mattia Frasca, Rosaria Volpe* \\ University of Catania, Department of Electrical, Electronics and Computer Engineering, viale \\ Andrea Doria 6, 95125 Catania, Italy \\ Email: rvolpe@dii.unict.it
}

\begin{abstract}
The energy consumption in cities is mostly satisfied by large scale fossil fuelled power plants that account for the major $\mathrm{CO} 2$ emissions. National and European policies point towards the adoption of proper energy measures aiming at reducing the emissions. In this direction, Distributed Energy Systems (DESs) are considered as an alternative to the traditional centralized supply, since they allow producing energy that may be distributed to consumers. Yet, the energy interactions occurring among consumers and installers of DESs are modeled within the framework of the complex networks, where nodes stand for consumers and/or potential producers and links symbolize their energy exchanges. The model is based on a cost analysis and minimizes the energy provided from the traditional power plant when a network of distributed energy systems is constituted within the urban territory. Moreover, the impact that the decentralized production has on the centralized supply in terms of cost of the energy delivered to consumers is evaluated. Results are discussed within an application to a numerical case study.
\end{abstract}

Keywords: Centralized Energy Supply, Complex Networks, Urban Areas, Distributed Energy Systems.

\section{INTRODUCTION}

Nowadays, the energy consumption in cities is mostly satisfied by fossil-fuelled power plants [1]. This large dependence on fossil fuels is responsible for a significant amount of $\mathrm{CO}_{2}$ emissions that are forecasted to increase in the near future [2]. In support of national and European policies pointing to the emissions reduction [3], local authorities are committed to address the transition towards more sustainable energy systems. In light of this, the introduction on urban territories of renewable based energy systems, such as the Distributed Energy Systems (DESs), is acknowledged as a cost-effective solution to move away from the fossil fuel dependence. Indeed, DESs offer, on one side, a clean alternative to the traditional energy production and, on the other side, afford to achieve the energy self-sufficiency of installers.

The insertion of distributed energy systems gives rise to the chance for installers to sell the produced energy and this possibility results, de facto, in a network of energy distribution [4].The insertion of DESs on urban territories also paves the way for the assessment of the impact that the decentralized production has on the centralized supply in terms of cost of the energy delivered to consumers.

This paper develops a methodology based on a cost analysis for the minimization of the energy supply from the traditional centralized power plants when a network of distributed energy systems is constituted within the urban territory.

The rest of the paper is structured as follows. Section 2 provides a literature review of the researches dealing with the mentioned issue. The model is introduced in Section 3 and a numerical case study is discussed in Section 4. Finally, Section 5 provides the conclusions.

\section{LITERATURE REVIEW}

Many scientific contributions deal with the issue of the insertion of DESs within urban areas. Yet, some authors cope with the cost analysis of the energy distribution by focusing on the design of DESs.

Along this issue, Yang et al. [5] present a mixed integer linear programming (MILP) model for the optimal design of DESs for the minimization of the annual cost for investing, maintaining and operating the system. The model is tested for a network of four buildings that may interact in order to exchange the own produced energy. Ren et al. [6] handle the same issue and combine the minimization of the energy costs with the minimization of the emissions. However, they do not consider the possible energy interactions among consumers, but rather they merely plan to sell the produced energy directly to the public utility grid. 
Other authors consider in their works the analysis of energy cost deriving from the supply mix of renewable and nonrenewable energy [7, 8 and 9].

In this direction, the paper of Stich et al. [10] provides a power supply optimization model for the cost-effective integration of renewable-based energy systems. Bandyopadhyay et al. [11] introduce a pinch analysis based method to find the optimum mix between the renewable and non-renewable energy based systems. Both contributions [10, 11] consider a potential grid extension, but not involve consumers in the distribution. Moreover, they do not insert sale scenarios in the cost analysis and mainly focus on the cost saving of the energy supply.

A more enlarged vision is proposed in the work of Rasid et al. [12]. The authors analyze the cost reduction that is achieved by replacing the power from fossil fuel-consuming grid generators with renewable-energy distributed generators. The fossil fuel cost saving is evaluated as the expected value due to the renewable output uncertainty.

The cited works mainly deal with either the cost analysis of DESs or of renewable and non-renewable energy mix. They do not evaluate the network of energy distribution that derives from the installation of DESs and, therefore, from the chance of consumers to sell the own produced energy. Nevertheless, among the proposed literature, the paper of Yang et al. [5] considers the sale among consumers. Anyway the study is conducted for a small network that cannot be compared to an urban neighborhood.

In addition to these considerations, the impact of those energy interactions on the minimization of the energy supply deriving from the centralized power plants is not considered.

The insertion of DESs requires a comprehensive evaluation of both the energy interactions that may occur among installers for the sale of the own produced energy and the impact that those interactions have on the traditional energy supply. Hence, proper mathematical models need to determine whether DESs are not only a green alternative to fossil energy, but also able to reduce the dependence on fossil-fuelled power plants.

The mathematical procedure introduced in this paper models the energy distribution among consumers as a complex network. In particular, nodes stand for consumers with both a given energy demand and the chance to install DESs. Links represent the connections that are responsible for the energy exchanges among nodes. The model is framed within a linear programming model and the objective function aims at finding the optimal configuration of the links that ensures the minimization of the energy provided from the power plant in terms of cost of the energy supply. A proper index is then introduced to evaluate the rate of connections between the power plant and each consumer after the installation of DESs.

\section{THE MATHEMATICAL MODEL}

The traditional energy distribution system is centralized, that is, the power plant provides energy to requesting consumers. This configuration changes when consumers install autonomous energy production systems. Indeed, the production of energy enables the possibility to distribute it. To model such a configuration of the energy exchanges, the complex network theory comes to the aid [13].

Two main elements are involved in the model; a set of $(N+1)$ nodes and a set of links. Nodes represent the $N$ consumers that may potentially install energy production systems, and the power plant, whilst links model the eventual energy exchanges among nodes. The power plant is labeled as $i=1$ and is hereinafter defined as central node to distinguish it from the other nodes of the network. Nodes may be identified as apartments, buildings or neighborhoods depending on the level of detail of the study. They are connected through a link on the ground of a threshold distance $d$, defined as the maximum admitted distance below which consumers may exchange energy. This criterion is not applied to the central node; indeed, each node is connected with the central node notwithstanding the distance.

Each node $i=2, \ldots, N+1$ is characterized by an energy demand $D_{i}$ and an eventual energy production $P_{i}$. The energy produced by each node aims primarily to satisfy the node own energy demand and, secondly, to distribute the energy excess to the connected nodes. In order to determine if a node requires energy to satisfy its energy demand or if it has energy to distribute, the energy surplus parameter is introduced as

$S_{i}=D_{i}-P_{i}, \forall i=2, \ldots, N+1$

The energy surplus parameter in Eq. (1) determines if the node $i$ belongs to the set of destination nodes, $D-S E T$, or to the set of source nodes, $S-S E T$. In particular,

- a destination node is characterized by a negative surplus, i.e. its energy demand exceeds its energy production and, in this case, the node needs to receive energy either from other nodes of the network or from the central node to meet its energy demand.

- a source node has, instead, a positive surplus; in this case, the energy production of the node exceeds the energy demand and, therefore, after the satisfaction of its own energy demand, the node distributes the energy excess to other nodes.

- $\quad$ Nil values of the energy surplus indicate that the energy production has totally equaled the energy demand of the node.

The network is described through a matricial representation and expressed in the $(N+1) \times(N+1)$ adjacency matrix

$$
A=\left[\begin{array}{ccccc}
0 & a_{1,2} & a_{1,3} & & a_{1, N+1} \\
a_{2,1} & 0 & a_{2,3} & & a_{2, N+1} \\
a_{3,1} & & & & a_{3, N+1} \\
& & & 0 & \\
a_{N+1,1} & a_{N+1,2} & & a_{N+1, N} & 0
\end{array}\right]
$$

The elements of the adjacency matrix indicate whether a link has been established or not. In particular, they assume the values

$$
a_{i j}=\left\{\begin{array}{l}
1, \text { if node } i \text { and node } j \text { are connected } \\
0, \text { otherwise }
\end{array}\right.
$$

The elements of the diagonal are zeros, i.e. self-connected nodes are not feasible. Moreover, the elements involving the central node are 1 , since each node is connected to it.

The elements of the adjacency matrix $A$ are updated to underline the direction of the energy surpluses; specifically, the exchanges follow the direction source node $\rightarrow$ destination node and not backwards. Therefore, $a_{i j}=1$ for the direction 
$i \rightarrow j$, and, vice versa, $a_{i j}=-1$ when energy flows from $j \rightarrow i$. Nil values remain unchanged.

The so defined network represents the starting topology of the energy distribution network, where nodes are characterized for being either destination or source nodes and links are established due to the distance criterion.

The central node always displays a nil energy demand, $D_{1}=0$, and is merely responsible for the supply. Obviously, when nodes do not install DESs, the central node is the sole supplier of energy. However, the insertion of DESs within the urban area configures a scenario of distribution among nodes that influences the supply of the central node. To study the impact of the DESs on the traditional supply, the problem is framed as a linear programming model with the objective to find the optimal energy exchanges among nodes for the minimization of the cost of the energy supply deriving from the central node. After the solving of the model, only those links that are effectively used for an energy exchange are maintained in the optimized network.

The objective function of the model is, therefore, expressed as

$\min \sum_{a_{1 j>0}} a_{1 j} e_{C N} X_{1 j}$

where $X_{1 j}$ are the energy flows that the central node distribute to each node $j$ of the network and $e_{C N}$ is the cost of the energy supply from the central node. Each node of the network has to respect the following energy balance.

$$
\sum_{j=1}^{N+1}\left(a_{i j} X_{i j}\right)=S_{i}, \forall i=1, \ldots,(N+1) \vee i \neq j
$$

As constrain, the sum of the cost of both the energy supply from the central node and the energy from the neighboring nodes should be lower than the traditional cost of the demand. This is formulated as

$$
\begin{aligned}
& a_{1 j} e_{C N} X_{1 j}+\sum_{i=1}^{N+1}\left(a_{i j} e_{\text {nodes }} X_{i j}\right) \leq e_{C N} D_{j}, \\
& \forall j=2, \ldots,(N+1) \vee j \in D-S E T
\end{aligned}
$$

where $e_{C N}$ is the unitary cost of energy sold from the central node, $e_{\text {nodes }}$ is the unitary cost of the energy deriving from the nodes of the network and $D-S E T$ is the set of the destination nodes.

Moreover, the model imposes that all energy flows are nonnegative.

$$
X_{i j}>0, \forall i, j=1, \ldots,(N+1), i \neq j
$$

The optimization problem firstly establishes the network of energy exchanges among the nodes of the network and secondly evaluates the energy provided by the central node. The measurement of the supply from the central node is conducted in a double way: on one side by calculating the amount of the energy supplied and, on the other side, by determining which links are effectively used for an energy exchange.
From a network design perspective it is interesting to figure out the impact of DESs on the supply of the central node. To the scope, the following index is introduced

$C N_{\text {index }}=\frac{\sum_{i=2}^{N+1} l i n k s_{1 \rightarrow i}}{N}$

The index is expressed as the ratio between the links exiting from the central node that are used for the exchange and the total links exiting from the central node in the traditional centralized network. The denominator of the index in Eq. (7) corresponds to the number of nodes, since each node is connected through a link to the central node in the traditional energy distribution network. The $C N_{\text {index }}$ varies within the interval $[0,1]$; in particular, $C N_{\text {index }}=0$ means that the central node does not supply any node of the network. This result means that the DESs installed on territory are able to entirely satisfy the energy demands of the nodes. Vice versa, for $C N_{\text {index }}=1$, the central node is still entirely responsible for the supply of energy to all nodes. This is true even when DESs are installed; indeed, the installed power is not sufficient to satisfy other nodes or is able to satisfy them only in part. Intermediate values indicate different rates of exploitation; however, as a general rule, the more small is $C N_{\text {index }}$, the more distributed is the network.

\section{NUMERICAL CASE STUDY AND DISCUSSION}

The mathematical model introduced in the previous Section is tested in a hypothetical urban territory of $1 \mathrm{~km}^{2}$ with $N=500$ randomly placed consumers connected on the ground of a threshold distance $d$, defined in the design stage. As an example, by setting $d=50 \mathrm{~m}$ as the distance of connection, the graphical representation of the case study area is exposed in Figure 1. In particular, Figure 1(a) displays the consumers as green squares and the admitted connections for the energy exchanges are indicated as red solid lines. In Figure 1(b) the connections with the central node, located in the bottom left of the space, are added and illustrated as blue dotted lines.

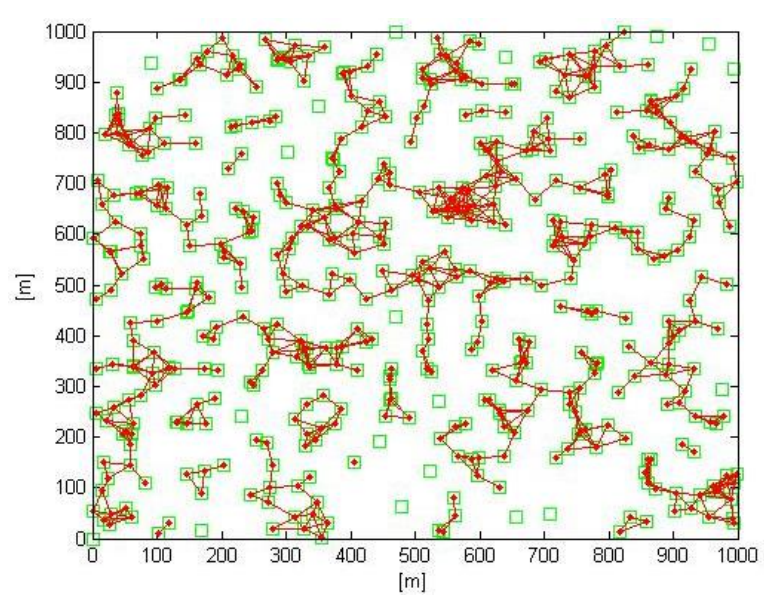

Figure 1. (a) Connected nodes on the ground of the distance threshold $d$ 


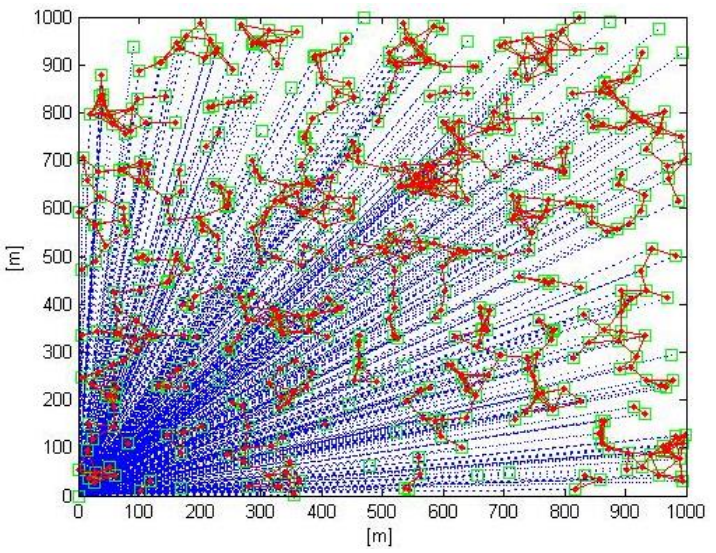

Figure 1. (b) Connected nodes on the ground of the distance threshold $d$ and connections with the central node

The energy distribution network of Figure 1(b) represents the starting point to run the model.

The energy demands of the nodes are varied within the interval [2 $\mathrm{MWh}, 7 \mathrm{MWh}$ ] according to a random uniform distribution [14]. Each node is considered as a potential installer of a DES and the total produced energy is expressed as a percentage of the total energy demand of the network, that is, the $50 \%$ of the energy production does not indicate that each node produces the $50 \%$ of its own energy demand, but rather that the sum of the energy produced by the installer nodes is the $50 \%$ of the total energy requirements of the network. The installation of DESs is randomly established.

The connections among nodes are allowed in line with four chosen values, namely for $d=50,100,150$ or $200 m$. Regarding the costs of the energy, they are assigned in accordance with Table 1 [15].

Table 1. Values of the energy cost parameters

\begin{tabular}{|c|c|}
\hline Energy costs & $c \mathrm{E} / k W h$ \\
\hline$e_{C N}$ & 0.17 \\
\hline$e_{\text {nodes }}$ & 0.10 \\
\hline
\end{tabular}

The model minimizes the energy provided from the central node and returns the values of the central node index $C N_{\text {index }}$. The values of the index $C N_{\text {index }}$ obtained by varying the percentages of energy production and in correspondence with four different threshold distances are shown in Figure 2.

The curves of Figure 2 have a similar trend independently of the considered threshold distance. The number of links that are responsible for the energy supply from the central node decreases for increasing values of the energy production percentages. More specifically, for percentages until the $20 \%$, the central node index assumes the value $C N_{\text {index }}=1$, i.e. the installation of DESs that account for the satisfaction of the $20 \%$ of the total energy demand of the network does not affect the supply from the central node. Indeed, within this range of installed power, nodes mainly satisfy in whole or in part their own demands and do not have sufficient exceeds to distribute. When increasing the percentage of installed power, the index $C N_{\text {index }}$ decreases. Nevertheless, in correspondence with the $100 \%$ of the energy production, i.e. for a percentage that potentially satisfies the whole demand of the network, the supply from the central node still remains significant. This is due to a twofold reason; on one side, the energy supply from the central node may be more convenient in comparison to the supply from the network (as in the case of several links exploited for the exchange and, therefore, a higher cost in fees) On the other side, the distance of connection poses limits to the distribution and, consequently, not all produced energy is effectively distributed.

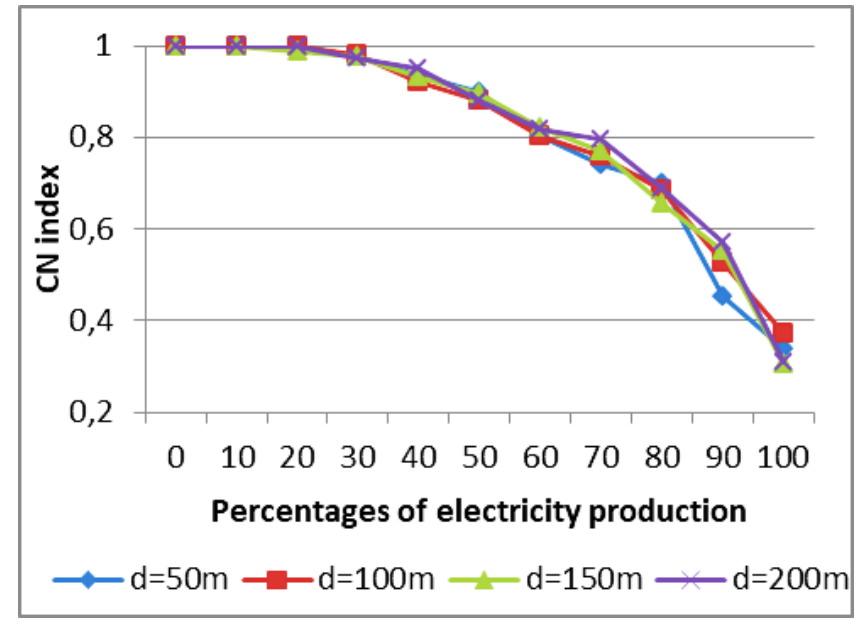

Figure 2. Trends of the $C N_{\text {index }}$ by varying the percentage of energy production in correspondence with four threshold distances

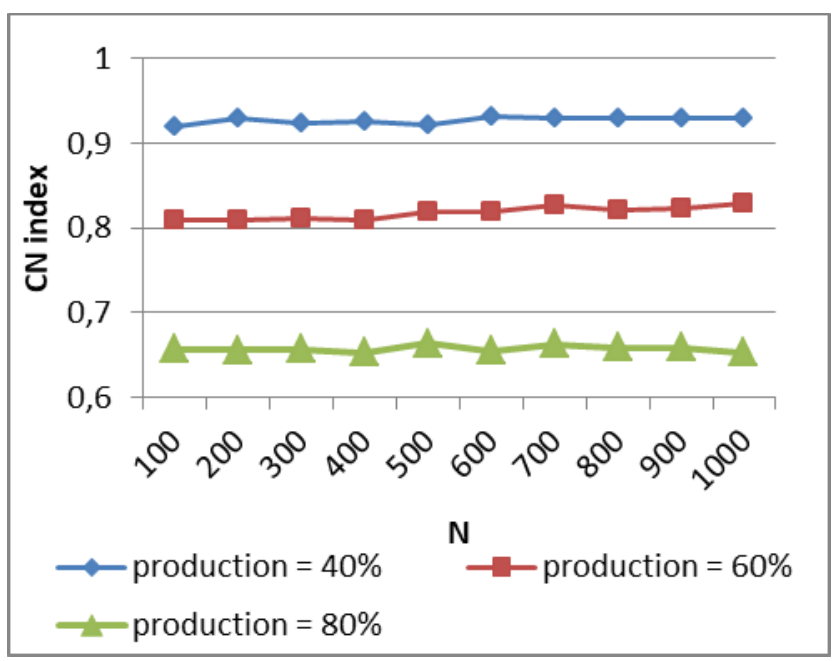

Figure 3. Trends of the $C N_{\text {index }}$ by varying the number of nodes at the distance $d=100 m$ for different values of the energy production percentages

The $C N_{\text {index }}$ index has been studied at varying the number of nodes of the network and the results are exhibited in Figure 3. As it can be observed from the figure, at a fixed energy production percentage the number of nodes does not impact on the supply from the central node, since the curves display an almost constant trend.

\section{CONCLUSIONS}

This paper proposes a cost analysis focused on the evaluation of the supply from the traditional fossil-fuelled power plant when distributed energy systems are inserted in 
the territory. Consumers are modeled as nodes characterized by an energy demand and an eventual energy production. The power plant is considered as a special node, with nil energy demand and labeled as central node to distinguish it from the other nodes. Each node gains a connection with the central node whilst the connections among the nodes of the network are permitted on the ground of a distance criterion for which two nodes are connected if their spatial distance is below a chosen threshold.

The model is constructed as a linear programming model with the aim to minimize the energy delivered from the central node in terms of cost of the energy supply. Moreover, to evaluate the impact that DESs have on the purchase from the power plant, a proper index is introduced to count the percentage of connections that are responsible for the supply from the central node in comparison to the traditional centralized configuration.

The model has been applied to a hypothetical urban area of $1 \mathrm{~km}^{2}$ with $N=500$ randomly placed consumers and the discussion has been conducted by varying the percentage of installers and the distance of connection among nodes.

The result permits to conclude that the insertion of DESs on territory allows achieving a reduction of supply from the grid. However, for the presented case study, this decrease is invariant with the distance of connection and with the number of nodes involved in the study.

\section{REFERENCES}

[1] IEA (2010). Electricity Information 2010, Technical Report, International Energy Agency, available at http://wds.iea.org/wds/pdf/documentation_EleInfo prelim 2010.pdf

[2] Fifth Assessment Report, AR5, available at http://www.ipcc.ch/report/ar57index.shtml

[3] Directive, 2009/28/EC of the European Parliament and of the Council, of 23 April 2009, on the promotion of the use of energy from renewable sources and amending and subsequently repealing Directives 2001/77/EC and 2003/30/E

[4] Fichera A., Frasca M., Volpe R. (2017). Complex networks for the integration of distributed energy systems in urban areas, Applied Energy, Vol. 193, pp. 336-345. DOI: 10.1016/j.apenergy.2017.02.065

[5] Yang Y., Zhang S., Xiao Y. (2015). Optimal design of distributed energy resource systems coupled with energy distribution network, Energy, Vol. 85, pp. 433-448. DOI: 10.1016/i.energy.2015.03.101

[6] Ren H., Zhou W., Nakagami K., Gao W., Wu Q. (2010). Multi-objective optimization for the operation of distributed energy systems considering economic and environmental aspects, Applied Energy, Vol. 27, pp. 36423651. DOI: 10.1016/j.apenergy.2010.06.013

[7] Cascio E.L., Borelli D., Devia F., Schenone C. (2017). Future distributed generation: An operational multiobjective optimization model for integrated small scale urban electrical, thermal and gas grids, Energy Conversion and Management, Vol. 143, pp. 348-359.

[8] Lazaretto A., Macor A., Mirandola A., Reini M. (1992). Analytical-symbolic method of thermoeconomic optimization of an energy recovery and cogeneration

system, ECOS '92, International Symposium, pp. 183-190, 1992.

[9] Manfren M., Caputo P., Costa G. (2011). Paradigm shift in urban energy systems through distributed generation: Methods and models, Applied Energy, Vol. 88, No. 4, pp. 1032-1048, 2011.

[10] Stich J., Hamacher T., Mueller M., Hesse C., Jossen A. (2016). Sustainable power supply options for large islands - A case study for Belitung Island, IEEE Innovative Smart Grid Technologies - ISGT Asia, Melbourne, Australia, Nov. 26th-Dec. 1st.

[11] Bandyopadhyay S., Desai N.B. (2016). Cost optimal energy sector planning: A pinch analysis approach, Journal of Cleaner Production, Vol. 136, pp. 246-253, 2016. DOI: 101.1016/j.clepro.2016.03.077

[12] Rasid M.M., Murata J., Takano H. (2017). Fossil fuel cost saving maximization: Optimal allocation and sizing of renewable-energy distributed generation units considering uncertainty via clonal differential evolution, Applied Thermal Engineering, Vol. 114, pp. 1434-1432. DOI: 10.1016/j.applthermaleng.2016.10.030

[13] Batty M. (2011). Cities as flows, cities of flows, Environmental and Planning B: Planning and Design, Vol. 38, pp. 195-196. DOI: $10.1016 / \mathrm{b} 3802 \mathrm{ed}$

[14] Fichera A., Volpe R., Frasca M. (2016). Assessment of the energy distribution in urban areas by using the framework of complex network theory, International Journal of Heat and Technology, Vol. 34, No. Sp. 2, pp. S430-S434. DOI: 10.18280/ijht.34S234

[15] Data available at

http://www.autorita.energia.it/allegati/docs/13/RappPolite cnicoRinn.pdf (in Italian)

\section{NOMENCLATURE}

$\mathrm{N}$

d

A

$a_{i j}$

$D_{i}$

$P_{i}$

$S_{i}$

$D-S E T$

$S-S E T$

$X_{1 j}$

$X_{i j}$

$e_{C N}$

$e_{\text {nodes }}$

$C N_{\text {index }}$

\section{Subscripts}

$i, j$

$\mathrm{CN}$

nodes number of nodes

distance of connection, $\mathrm{m}$ adjacency matrix elements of the adjacency matrix energy demand of node $i, \mathrm{kWh}$ energy production of node $i, \mathrm{kWh}$ energy surplus of node $i, \mathrm{kWh}$ set of destination nodes set of source nodes energy flows exiting from the central node and pointing to node $j, \mathrm{kWh}$ energy flows exiting from node $i$ and pointing to node $\mathrm{j}, \mathrm{kWh}$ unitary cost of the energy from the central node, $\mathrm{c} € / \mathrm{kWh}$ unitary cost of the energy sold from the nodes of the network, $c € / \mathrm{kWh}$ central node index

nodes of the network, with $i, j=2, \ldots, N+1$ central node nodes 